\title{
Correction to: Modelling cellular spreading and emergence of motility in the presence of curved membrane proteins and active cytoskeleton forces
}

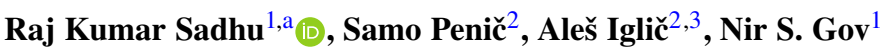

${ }^{1}$ Department of Chemical and Biological Physics, Weizmann Institute, Rehovot, Israel

2 Laboratory of Physics, Faculty of Electrical Engineering, University of Ljubljana, Ljubljana, Slovenia

${ }^{3}$ Laboratory of Clinical Biophysics, Faculty of Medicine, University of Ljubljana, Ljubljana, Slovenia

(C) The Author(s), under exclusive licence to Società Italiana di Fisica and Springer-Verlag GmbH Germany, part of Springer Nature 2022

\section{Correction to: Eur. Phys. J. Plus (2021) 136:495}

https://doi.org/10.1140/epjp/s13360-021-01433-9

In this article, the following addition has been made to the acknowledgments:

"N.S.G. acknowledges support by the Ben May Center for Theory and Computation."

The original article has been corrected.

The original article can be found online at https://doi.org/10.1140/epjp/s13360-021-01433-9.

a e-mail: raj-kumar.sadhu@weizmann.ac.il (corresponding author) 